\title{
Suicidios en mayores de 65 años en la provincia de Badajoz: análisis médico legal.
}

Suicide in the over 65's in the county of Badajoz: forensic analysis.

M. Casado Blanco ${ }^{1}$

\section{RESUMEN}

En el presente trabajo se dan a conocer los datos reales del suicidio consumado en un ámbito territorial específico, como es la provincia de Badajoz, durante un período de 5 años. Analizamos una serie de variables en cada uno de los casos de suicidio consumado que podían influir en este tipo de conducta. Hemos tratado de obtener conclusiones válidas extrapolables a la población suicida en general, mayor de 65 años. Con ello se pretende efectuar una mínima aportación a una línea de investigación que tiene como finalidad conocer mejor el tema del suicidio para poder actuar sobre su prevención.

Palabras clave: Suicidio consumado. Mayores de 65 años. Medicina legal

\section{ABSTRACT}

The present paper presents data of the suicides committed in a specific territorial field, the province of Badajoz, during a five-year period. In each case of successful suicide, a series of variables which could influence suicidal conduct are analysed. We have tried to obtain valid conclusions which we could extrapolate to the suicidal population in general aged over 65. Our intention is to make a small contribution to a line of research which aims at a better nderstanding of suicide so as to facilitate preventive action.

Key words: suicide, over-65's, forensic medicine.

Correspondencia: Mariano Casado Blanco. Facultad de Medicina Badajoz. Área de Medicina Legal y Forense. Avda. de Elvas s/n. 06071. Badajoz.

1 Doctor en Medicina. Médico Forense. Profesor Asociado de Medicina Legal, Universidad de Extremadura. 


\section{INTRODUCCIÓN:}

El suicidio es una forma relativamente frecuente de muerte y constituye un fenómeno social de gran importancia y trascendencia. Sin duda alguna, su estudio, valoración y prevención es una responsabilidad que compete a todos los niveles sanitarios. En principio, aunque esta afirmación pueda resultar clara, no parecen existir todos los argumentos necesarios para enfrentarse a ellos $[1,2]$. Por otra parte, se contabiliza que aproximadamente un $25 \%$ de las personas que hacen una tentativa de suicidio no entran en contacto con dicho sistema sanitario [3].

El conocimiento de los factores de riesgo de suicidio, tanto de tipo demográfico como clínicos, resultan a todas luces útiles para poder estimar el grado de peligro. Aunque este método resulta apropiado para conocer esta realidad, no podemos olvidar que está lejos de ser seguro e indubitable.

No existe duda alguna acerca de la necesidad de una colaboración multidisciplinaria para el estudio del suicidio, dada la complejidad de esta conducta y su expresión de una causalidad múltiple [4].

En todo este entramado, la figura del médico generalista y del psiquiatra resultan clave en la detección precoz del riesgo de suicidio. Numerosos estudios ponen de manifiesto que aproximadamente el $50 \%$ de los pacientes que consuman el acto suicida, comunicaron en algún momento al médico de "cabecera" y/o psiquiatra, su intención en fechas próximas a la consumación.

Orientado por determinados factores de riesgo, que trataremos de analizar, el médico debe saber detectar estos casos y en los supuestos de no encontrarse capacitado para su seguimiento y control incluso en aquellos en los que pudieran parecer insignificante el riesgo, deberá realizar su derivación hacia servicios psiquiátricos, ya que los factores protectores están o deben estar relacionados con esta atención especializada [5].

La intención de nuestro estudio es llegar a conocer estos posibles factores de riesgo que inciden en la población mayor de 65 años, mediante un estudio retrospectivo de tipo epidemiológico descriptivo, considerando esta edad como punto de inflexión en el devenir de toda persona y por constituir un problema de considerable importancia, debido a su magnitud [6].

En líneas generales, diferentes investigaciones sobre el tema vienen a poner en evidencia diferencias cualitativas y cuantitativas, según los países, e incluso dentro de cada país, según las zonas geográficas. Así, a modo de ejemplo y de forma general, en nuestro país resulta más frecuente el suicidio en la población juvenil y entre las mujeres [I], mientras que en Estados Unidos es tres veces más frecuente en hombres, especialmente en edades superiores a los 50 años [7].

Aparte de estos factores, aparecen otras posibles circunstancias desencadenantes del acto suicida. En relación al grupo de edad que nos proponemos analizar, los antecedentes psiquiátricos resultan muy llamativos en los numerosos estudios revisados $[1,7,8,9,10,1 \mathrm{I}]$, en especial la depresión y los cuadros esquizofrénicos.

No podemos olvidar la importancia de los acontecimientos vitales, en especial aquellos que representan pérdidas afectivas importantes. Finalmente, existe lo que Corbella [I] llamó "suicidio balance", cuando alguien decide que la vida ya no tiene valor para él, luego de efectuar un balance acerca de su existencia.

\section{MATERIAL Y MÉTODO:}

Se revisan las estadísticas de autopsias realizadas por muertes violentas en los diferentes Partidos Judiciales de la provincia de Badajoz, durante un período de cinco años (1990-1995). Durante el citado período contaba con una población en torno a los 657.000 habitantes, de los cuales 122.225 habitantes corresponden a Badajoz capital, según se recoge del censo de 1991. 
Consideramos que la fuente es extremadamente fiable, por cuanto los datos médico-forenses deben constituir una excelente muestra para conocer la realidad suicida [12].

De estos datos, se analizaron los casos calificados por parte del Médico Forense como suicidios, en cada uno de ellos se cumplimentó un protocolo, en el cual se recogía información acerca de diferentes datos relativos a la filiación (sexo, estado civil, edad, ocupación), antecedentes personales (asociación con factores económicos, emocionales, lúdicos, de tratamiento psiquiátrico o de enfermedades terminales) y los relativos al propio acto suicida (lugar del suicidio, horario, mes, método empleado, existencia de alguna nota amenazante o explicativa del motivo o motivos).

Basándonos en estos objetivos se utilizaron desde el punto de vista estadístico el test chicuadrado de Pearson al tratarse de variables cualitativas, para una vez procesada y tratada la información, nos ayudase a conocer una realidad o fenómeno socio-médico-cultural, de tanta transcendencia como resulta ser el acto suicida.

Los datos recogidos se complementaron con otros obtenidos a nivel familiar e incluso mediante informes clínicos encontrados en el propio domicilio del suicida o bien en historiales clínicos obrantes en los archivos de los diferentes Centros de Salud y/o en Hospitales. De esta forma se completaron todos los datos referentes al protocolo propuesto y se establecieron como resultados de significancia el nivel $p<0,05$. Todo el estudio se realizó utilizando el programa Statview de Macintosh.

\section{RESULTADOS:}

Del análisis general de los datos obtenidos durante el período estudiado, encontramos 290 casos de suicidios consumados, de los que 110 casos corresponden a sujetos con edad superior a los 65 años, representando un 37,93\% del total.

La distribución del número de casos referida a los años analizados ofrece valores muy similares, a excepción de los años 1993 y 1994 que representaron un 48, I \% (53 casos). A pesar de ello, las oscilaciones anuales, tanto en lo que se refiere a los varones como a las mujeres, transcurren paralelas en casi todos los años, es decir cuando aumenta el número de suicidios consumados lo hace de forma similar para ambos sexos y del mismo modo,

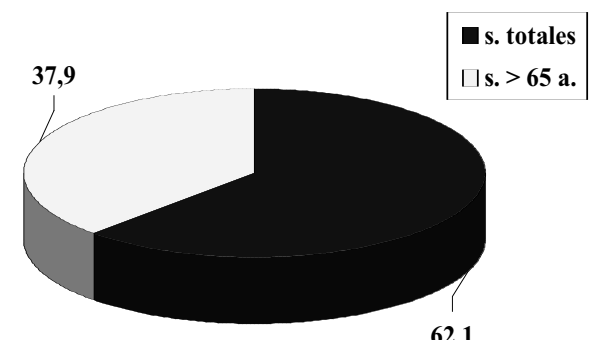
al disminuir el número total de aquellos también lo hace proporcionalmente en ambos sexos.

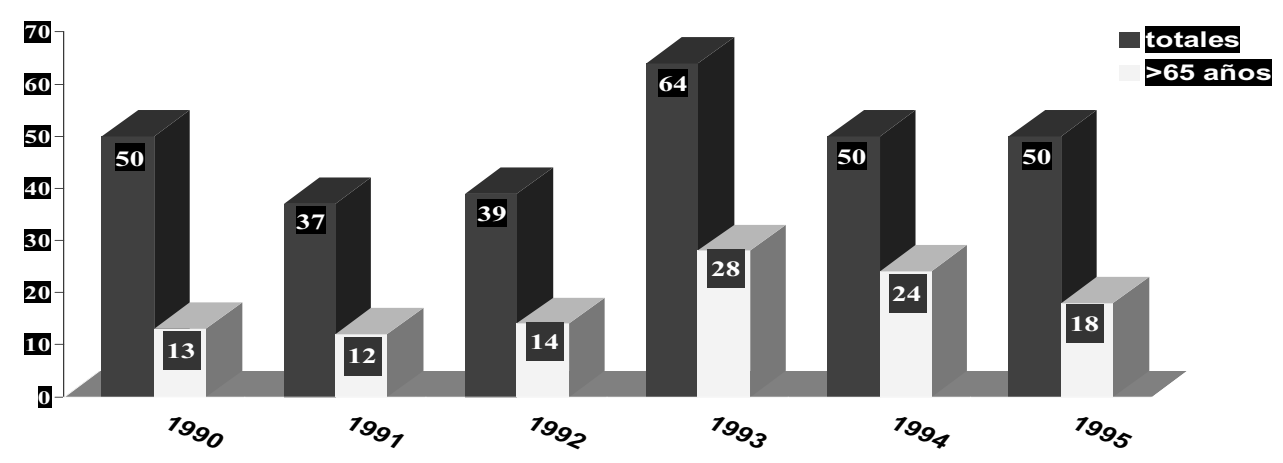

$\triangle$ NÚMERO DE SUICIDIOS POR AÑOS. 
Al analizar los días de la semana en los que se realizan actos suicidas, no se encuentran diferencias significativas, presentando una distribución similar, en torno al 15-19\%, exceptuados los fines de semana (sábados y domingos) en los que la incidencia es notoriamente inferior (8,5\%).

Cuando se estudia la distribución por meses y por estaciones, se observan pequeñas oscilaciones mensuales con resultados no significativos, con una tasa de actos suicidas más frecuentes en los meses que comprenden la primavera (abril, mayo y junio), con un 33,6\% del total.

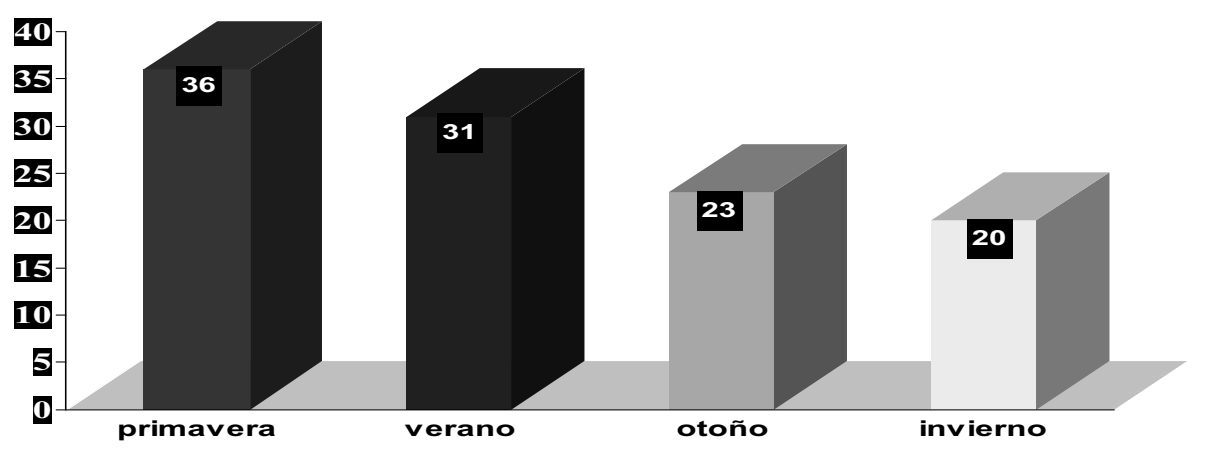

A NÚMERO DE SUICIDIOS POR ESTACIONES.

En relación al sexo, el resultado es concluyente con un claro predominio de los hombres, representado por un $83,6 \%$ (92 casos) y un 16,4\% en las mujeres (I 8 casos), lo que representa una proporción de 5: 1 .

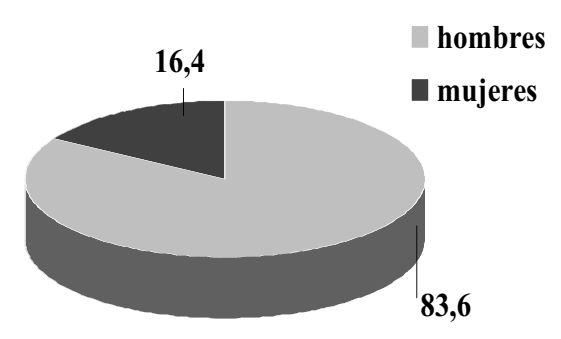

A NÚMERO DE SUICIDIOS POR SEXO.

Al estudiar estos datos atendiendo a los diferentes grupos de edad en que hemos dividido a la población, se aprecia que en el sexo masculino aparecen dos picos de edad llamativos, uno en el período entre los 64-67 años $(31,25 \%)$ y otro a partir de los 79 años (32,8\%). En cambio en las mujeres suelen aparecer cifras similares en todos los períodos, excepto entre los 68-7l años, en el que se da la mayor incidencia.

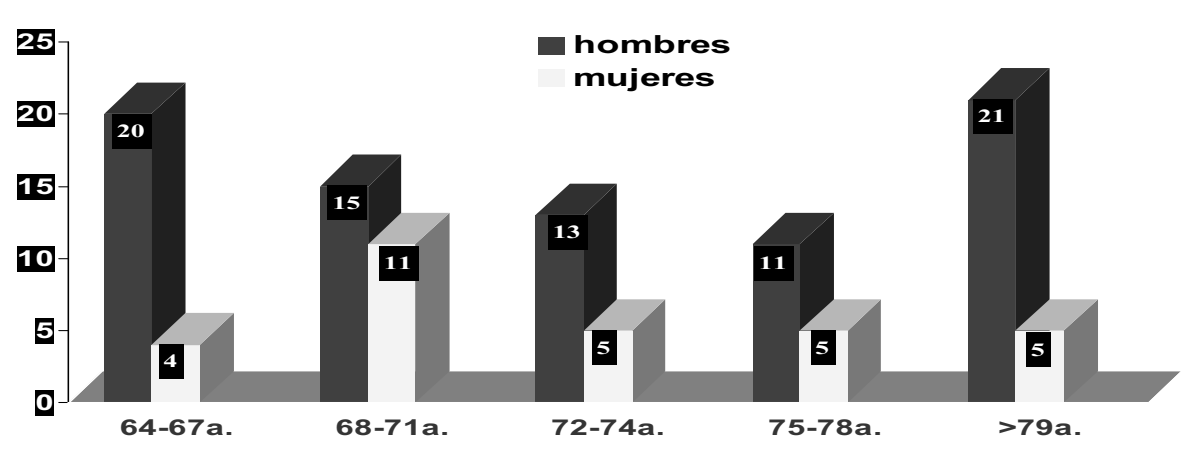

$\triangle$ NÚMERO DE SUICIDIOS POR SEXO Y EDAD. 
En lo que hace referencia al estado civil de la muestra, 54 casos corresponden a personas casadas (49\%), 36 casos fueron viudos (32,7\%), 18 casos solteros $(16,3 \%)$ y 2 casos separados o divorciados.

Analizado el lugar de procedencia y teniendo en cuenta las características de la provincia de Badajoz, donde prevalecen los núcleos rurales, se establecieron tres grupos de poblaciones atendiendo al número de habitantes. La mayor incidencia de suicidios corresponde a los núcleos de población inferior a los diez mil habitantes, representando el 69\% de los casos; un 10,9\% se produjo en poblaciones de hasta veinticinco mil habitantes y un $20 \%$ para las restantes, es decir, aquéllas cuyo número de habitantes supera los veinticinco mil.

Por lo que se refiere al método para consumar el suicidio, el más utilizado fue la ahorcadura con 65 casos, lo que supone un 59\%, seguida, a gran distancia de la sumersión (22,7\%) con 25 casos. La ahorcadura fue el método de elección entre los hombres y también para las mujeres. Referente al mecanismo de sumersión, el lugar que más frecuentemente se utiliza es el río o pantano con algo más del $86 \%$ de los casos, seguido del pozo de la casa (10\%).

El $81,8 \%$ de los suicidios de la muestra se llevó a cabo en el propio domicilio, siendo este efectuado en lugares dispares y variados, aunque al tratarse de casas rurales la mayoría ocurren en corrales, cuadras, patios...

La asociación con consu-

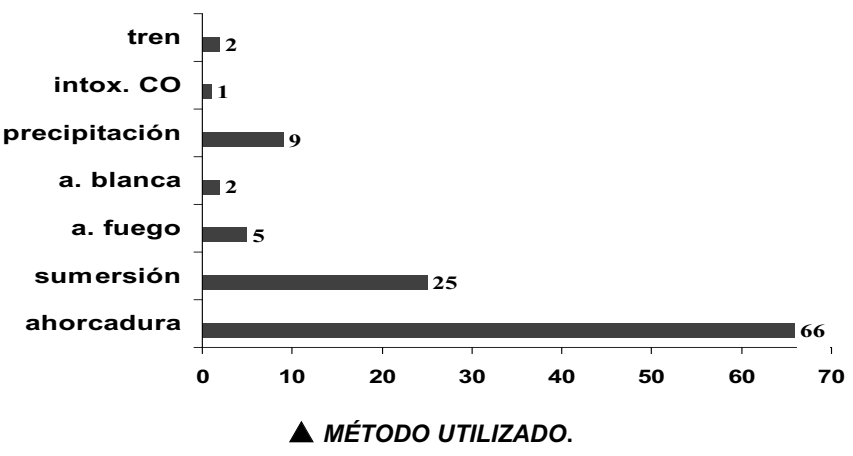
mo de alcohol, con o sin dependencia, como causa única desencadenante del acto, fue negativa en el $99 \%$ de los casos. $\mathrm{Si}$ bien es cierto que el cortejo del acto implica en algunas ocasiones el previo consumo de alcohol, esta circunstancia no pudo comprobarse al no haberse practicado determinaciones de alcoholemia de forma sistemática.

La presencia de enfermedades somáticas como factor principal, fue detectada en el $9 \%$ de los casos, tratándose generalmente de procesos crónicos de diferentes etiología (enfermedades degenerativas articulares, enfermedades crónicas pulmonares, cerebrales...), lo que suponen cifras ligeramente inferiores a otros estudios [13, I 4].

Las patologías psiquiátricas conocidas aparecieron en el 36,3\% como única causa determinante. Dentro de estas, los cuadros depresivos resultaron ser los más frecuentes y

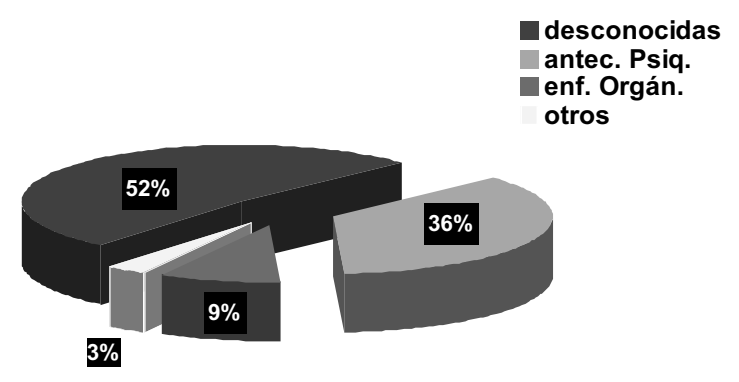

A CAUSAS/MOTIVOS DE SUICIDIOS. sobre todo, en los sujetos varones y de estado civil viudo. Resulta llamativo que en el $51 \%$ de la muestra no se determinó ninguna causa conocida.

Un 10\% (I I casos) había realizado intentos de suicidio anteriormente al acto consumado y así mismo, un । I,8\% ( 3 casos) dejó algún tipo de documento explicativo o justificativo de dicha acción. 


\section{DISCUSIÓN:}

Como primer dato interesante y digno de estimación, es la tasa de suicidios consumados en la población mayor de 65 años de edad, en la provincia de Badajoz, que siguiendo a lo establecido por la O.M.S., esta se fija en función del número de actos suicidas/cien mil habitantes. Por tanto, nuestros resultados se pueden considerar con una tasa media-alta, al hallar valores de 16,7, lo que representa un porcentaje en torno al 38\% del total de actos consumados, a la vez que resulta una tasa muy superior a la encontrada en la población general de nuestra provincia, hecho que corrobora la existencia de una correlación entre suicidio y edad.

Se mantiene el sexo, al igual que en la totalidad de otros trabajos similares, marcadamente superior en varones, e incluso algo superior en relación a otros trabajos semejantes $[17,18,19]$, con una proporción significativa de 5:1. Esta proporción se mantiene constante en todos los grupos de edad analizados. Respecto al estado civil, los grupos que encontramos con un mayor índice de suicidios consumados, a diferencia de otros trabajos similares [29,30], son los casados sobre todo en los hombres, en una proporción de $4: 1$, seguido del estado viudo, que aunque se mantiene más frecuente en el sexo masculino, la proporción, con relación a las mujeres, se reduce considerablemente respecto a las viudas, representando una proporción de 2: I .

Estos datos no siguen la tendencia de considerar el matrimonio como un factor protector, sino que quizás con el paso de los años aparecerían factores propios como desacuerdos, desaveniencias o dificultades matrimoniales que podrían actuar como acontecimientos vitales o estresantes. En el caso del estado civil viudo, factores como la posible soledad, aislamiento, falta de perspectiva de futuro, sin olvidar las patologías somáticas, constituirían factores marcadamente desencadenantes de la realización de dichos actos. Además resulta interesante destacar que los grupos de edad no siguen el mismo perfil en el caso de hombres y de mujeres, encontrando en el caso de los hombres el mayor porcentaje de actos suicidas entre los 65-67 años y sobre todo a partir de los 79 años, donde, además, la mayoría son viudos, lo que se podría traducir indicando que el hombre anciano puede tener menos recursos vitales para afrontar y resistir una vida solitaria.

Analizando los posibles motivos o causas que llevan a tomar tal decisión, encontramos una gran proporción de casos en que la mayor parte de los suicidios consumados tienen como protagonistas a personas mayores que padecen algún tipo de alteración psíquica identificable, que en nuestro caso daría explicación a más del 36\%, cuestión esta importante, pues son patologías que pueden ser tratadas y sobre las que se puede intervenir de forma preventiva. Las alteraciones psicopatológicas que más frecuentemente encontramos son los trastornos de tipo afectivo, dato por otra parte de más reconocido y estimado en torno al I5\% [20]. Nuestra muestra presenta una proporción del 36\% (40 casos) que seguía un control y tratamiento específico para los episodios depresivos y de estos, destacan una mayoría del sexo femenino y de estado civil viuda.

Otro dato interesante, y diferenciador de otros trabajos [21,22], es la escasa relación, directa y única de causa-efecto, que encontramos en nuestra muestra de dependencia a sustancias psicoactivas, ya se trate de alcohol o drogas, y el acto suicida $[26,27,28]$, a pesar de que algunos autores indican que esta relación aumenta el riesgo de suicidio hasta cinco veces [3 I].

En cuanto a los métodos utilizados, los resultados parecen orientar que teniendo en cuenta determinados factores, como pudieran ser el medio rural, disponibilidad, accesibilidad..., el mecanismo más empleado, y además con gran diferencia, es la ahorcadura, dato que ya fue resaltado en el trabajo realizado, en nuestra zona, por Porras Obeso [29], en el período 1978- 1985 y de otros similares efectuados en diferentes zonas de España. [19,23,24].

La época del año y día de la semana preferente de consumación del acto suicida, en nuestra serie y a diferencia de otros trabajos, [12,19,25] encontramos una mayor incidencia durante los meses de primavera (abril, mayo y junio), mientras que los días de la semana son los sábados y domingos. 


\section{CONCLUSIONES:}

Como conclusiones, se puede indicar que los indicadores de riesgo obtenidos en el presente estudio, han de tomarse con las oportunas reservas, pero por supuesto que constituyen una aproximación al fenómeno del comportamiento suicida, de los mayores de 65 años, en la provincia de Badajoz.

Por otro lado, parece fundamental que en la formación de los diferentes profesionales, se ha de prestar la atención que merece el comportamiento suicida, no solo en la época de la senectud, sino a lo largo de todo el ciclo vital.

Todo esto teniendo en cuenta que los actos suicidas consumados se constituyen con demasiada frecuencia como la única solución a determinados problemas o cuestiones, que sufren y/o padecen los mayores de 65 años de edad, y por ello los diferentes colectivos profesionales tienen la responsabilidad de saber reconocer el riesgo real de suicidio e intervenir con garantías sobre esta trágica realidad.

\section{BIBLIOGRAFIA:}

I.- Corbella Corbella J.: El Suicidio. En Gisbert Calabuig J.A.: Medicina Legal y Toxicología. Salvat Editores, 1991: 262-268.

2.- Ugaldelobo J., Castro F.J.: El suicidio en los hospitales generales de Costa Rica (Sistema Hospitalario Nacional, 1980-1990). Medicina Legal de Costa Rica, 1992: 9-14.

3.- Kennedy, PF, Kreitman N.,: "An epidemiological survey of parasuicide (attempted suicide) in general practice" . Br. J. Psychiatry, 1973; 123: 23-24.

4.- Sarró B., Bossa M., Carol M., et al.: Suicidio. Monografías Médicas Jano, 1988; 2(9): 5-7.

5.- Appleby L.: Suicide in psychiatric patients: risk and prevention. Br. J. Psychiatry. 1992; 161: 749-758.

6.- Díaz Suárez J., Sáiz Martínez P.A., Bousoño García M., Bobes García J.: Prevención de las conductas suicidas y parasuicidas. Concepto y clasificación de las conductas suicidas. Ed. Masson, Barcelona. 1997: 13-33.

7.- Kaplan H., Sadock B. Compendio de Psiquiatría. Salvat Editores, Barcelona. 1987: 1305-1309.

8.- Toro R.J., Yepes L.E.: Fundamentos de medicina: Psiquiatría; MedeIlín: Corporación para Investigaciones Biológicas (CIB), 1986.

9.- Shrun Johnston A.: Fire and Suicide: $A$ three study of self inmolation Deaths. Journal of Forensic Sciences. 1980; 37 (I): 209-22I. 10.- Herman SP, Schomalter J.,: Depresión y Suicidio en la niñez. Tribuna Médica, 1982: 7-10.

II.- Bluglers R., Bowden P.: Principies and Practice of Forensic Psychiatry. Churchill Livingstone 1990: 597-610.

I2.- Irigoyen I., Vilaspasa I., Alegret M., Gabriel C.: Características del suicidio consumado en la población rural. Psiquiatría Pública. 1997;9 (2):105-109.

13.- Hjortso T.: Suicide in relation to somatic illness and complications. Crísis. 1987; 8-2: 125-137.

14.- Sáiz J.L., López Ibor J.M., Benítez F., Cañas F.: Suicide dans I'Hôpital General. En: Soubrier JP, Vedrinne J., ed. Depression et suicide. París, Pergamon Press, 1981: 489-495.

15.- Deshaies G.: Psychologie du suicide. París. Presses Univ. France, 1947.
16.- Aso Escario J., Navarro Celma J.A., Cobo Plana J.A.: El suicidio consumado en Zaragoza (1988-199I). Aspectos epidemiológicos y perfiles suicidas. Actas Luso Esp. Neurol Psiquiatr.1993; 21(3):65-71.

17.- Martí Amengual G.: El suicidio consumado en las Islas Baleares. Tesis Doctoral. Universidad de Barcelona, 1982.

18.- Domingo Carrasco C.: Estudio del suicidio consumado en el partido Judicial de Castellón (1982-1986). Memoria de Licenciatura. Universidad de Alicante, 1987.

19.- Romero Cervilla C., Balanza Alonso de Medina E., Alarcón Jiménez F., Brell González JL.: El sucidio en el Partido Judicial de Sevilla en el quinquenio 1990-1994. Cuad. Med. For. 1996, (3):39-45.

20.- NIH. Consensus Conference: Diagnosis and treatment of depression in late life. JAMA, 1992; 268: 1018-1024.

2I.- Pamblanco Lillo E., Navarro López MI, Martí Lloret JB, Rodes Lloret F.: Suicidio y alcohol: comentario acerca de un caso. Rev. Esp. de Medicina Legal.1990; 62-63: 15-17.

22.- Grau M., Sierra E.: Suicidio y alcohol. Monografías Jano,1988;2(9):56-58.

23.- Rodado JM,. García JA., De Francisco P, Menéndez JA: El suicidio en Segovia (1992-1994). Rev. Esp. Neuropsq. 1997; 18 (62):25I-269.

24.- Franco Martín MA., Monforte Porto JA., Fernández Rojo C., Díez Boizas ].: Intentos de suicidio en Zamora: estudio retrospectivo de los años 1989-1994. Psiquis.1997; 18(5): 208-217.

25.- Rodes Lloret F., Pamblanco Lillo E., Martí Lloret JB., Navarro López MI.: El suicidio en la tercera edad. Rev Esp. Med. Leg. 1990; 6263:7-13.

26.- Barraclough B., Brunch J., Nelson B., Sainsbury P.: A lundred cases of suicide: clinical aspects. Br. J. Psychiatry.1974; 125:355-373.

27.- Beskow J.: Suicide and mental disorder in Swedishmen. Acta Psychiatr Scand., 1979: (227): I-138.

28.- Alonso Fernández F:: Alcoholdependencia. Personalidad del alcohólico. $3^{a}$ edición. Masson-Salvat. Madrid. 1992: 83-90.

29.- Durkheim E.: El suicidio. Ed. Akal. Madrid, 1985.

30.- Simonin C.: Medicina Legal judicial (legislación y jurisprudencia españolas). Ed. Jims. Barcelona, 1982: 772-776.

31.- Ghost T., Víctor B.S.: Suicidio. Ed. Ancora, S.A. Tratado de Psiquiatría. The American Psychiatric Press. Barcelona 1996: I313-1334. 